\title{
Mejora del estado nutricional en los participantes del proyecto “Ceupropsf”, 2017-2019
}

\author{
Improvement of the Nutritional Status in the participants of the "Ceupropsf" \\ Project, 2017-2019
}

Agudo Gonzabay Brigida Maritza*

Universidad Técnica de Machala

bagudo@utmachala.edu.ec

Machala - Ecuador

Arévalo Córdova Tania Diciana

Universidad Técnica de Machala

tarevalo_est@utmachala.edu.ec

Machala - Ecuador

Aguilera López Gerardo De Las Mercedes

Hospital Básico 1B1 El Oro

aguilera-gerardo@hotmail.es

Machala - Ecuador.

Aguilera Agudo Jonathan Gerardo

Universidad Estatal de Guayaquil/ Jonathan-aguilera@ hotmail.com

Machala - Ecuador.

\section{Resumen}

El objetivo del trabajo fue determinar la mejora del estado nutricional en los participantes del proyecto Ceupropsf. Se realizó una investigación descriptiva, observacional, no experimental, cualitativa, diseño prospectivo. Los resultados fueron: al inicio del proyecto, el $48 \%$ tenían sobrepeso, $21 \%$ peso normal, $19 \%$ obesidad moderada, $9 \%$ obesidad severa y $2 \%$ obesidad mórbida y bajo peso; al finalizar el proyecto, el $46 \%$ tuvieron sobrepeso, $34 \%$ peso normal, $15 \%$ obesidad moderada, $7 \%$ obesidad severa. En conclusión, se observó una mejora en el estado nutricional de los participantes al finalizar el proyecto, al disminuir los pacientes obesidad severa, moderada y sobrepeso.

Palabras clave: Estado nutricional, enfermedades crónicas no transmisibles, sobrepeso, obesidad, Ceupropsf. 


\section{Abstract}

The objective of the study was to determine the improvement of the nutritional status in the participants of the Ceupropsf project. A descriptive, observational, non-experimental, qualitative, prospective design research was carried out. The results were: at the beginning of the project, $48 \%$ were overweight, $21 \%$ were normal weight, $19 \%$ were moderately obese, $9 \%$ were severely obese, and $2 \%$ were morbidly obese and underweight; At the end of the project, $46 \%$ were overweight, $34 \%$ were normal weight, $15 \%$ were moderately obese, and $7 \%$ were severely obese. In conclusion, an improvement in the nutritional status of the participants was observed at the end of the project, as the patients decreased severe, moderate and overweight obesity.

Keywords: Nutritional status, chronic non-communicable diseases, overweight, obesity, Ceupropsf.

\section{Introducción}

La obesidad es una enfermedad crónica caracterizada por aumento del tejido adiposo, causado por el desbalance entre la energía ingerida en los alimentos y la energía eliminada caracterizada por un alto grado de inflamación y oxidación (Nafxiel y Alcázar, 2011). La obesidad se caracteriza por un mayor contenido de grasa corporal y dependiendo de su magnitud y de su ubicación topográfica va a determinar riesgos de salud que limitan las expectativas y calidad de vida. En adultos, la obesidad se clasifica de acuerdo al IMC por la buena correlación que presenta este indicador con la grasa corporal y riesgo para la salud a nivel poblacional (Moreno, 2012).

La obesidad se considera uno de los principales factores de riesgo cardiometabólico, pudiéndose medir mediante el Índice de Masa Corporal (IMC) (Hernández et al, 2018; Domínguez et al, 2017) El IMC o índice de Quetelet, es una de las medidas antropométricas más utilizadas en la práctica clínica diaria, y se calcula dividiendo el peso corporal de una persona, expresado en kilogramos, por su altura, expresada en metros y elevada al cuadrado (García et al., 2018).

En el año 2016, se estimó que el número de personas obesas en el mundo era de más de 300 millones, distribuidos por todo el mundo, teniendo mayor prevalencia en los países desarrollados o en vías de desarrollo (García y Creus, 2016).

Las enfermedades crónicas no transmisibles constituyen en la actualidad uno de los mayores retos que enfrentan los sistemas de salud a nivel mundial, debido al gran número de casos afectados, las tasas de incidencia en aumento, su gradual contribución a la mortalidad general, ser la causa más frecuente de discapacidad, costo elevado de la hospitalización, tratamiento médico y rehabilitación (Ángel y Valdés 2016; Serra et al, 2018).

Las enfermedades no transmisibles anualmente causan el $71 \%$ de las muertes a nivel mundial, en el rango de edad de 30 a 59 años, han fallecido 15 millones de personas por esta causa. Las enfermedades cardiovasculares constituyen la mayoría de las muertes por 
enfermedades no transmisibles (17,9 millones cada año), seguidas del cáncer ( 9,0 millones), las enfermedades respiratorias (3,9 millones) y la diabetes (1,6 millones) (Organización Mundial de la Salud (OMS), 2018).

Entre el 20\% y 35\% de la población adulta de América Latina y el Caribe tiene hipertensión (Organización Panamericana de la Salud (OPS), 2017). Aproximadamente 62 millones de personas tienen Diabetes tipo 2 en las Américas (OPS, 2018). En el Ecuador, en el 2017, según el Instituto Nacional de Estadísticas y Censos del Ecuador, INEC (2018), se dieron 16.585 egresos hospitalarios por Diabetes mellitus y 5776 por Hipertensión Arterial. En la provincia de El Oro, existieron 1012 por Diabetes Mellitus y 495 por Hipertensión Arterial.

\section{Antecedentes}

En la Parroquia la Providencia existe un número considerable de personas que padecen de enfermedades crónicas no transmisibles como Hipertensión Arterial y Diabetes Mellitus, por lo que la carrera de Ciencias Médicas de la Unidad Académica de Ciencias Químicas y de la Salud, en conjunto con la carrera de Ingeniería en Sistemas de la Unidad Académica de Ingeniería Civil de la Universidad Técnica de Machala, instauraron el proyecto de vinculación con la sociedad: Centro Universitario de Promoción y Prevención de la Salud Familiar CEUPROPSF.

Este proyecto de vinculación a la comunidad, realizó diferentes estrategias de promoción y prevención de la salud, para atender problemas como: inadecuados hábitos alimenticios, sedentarismo, falta de medidas de higiene saludables e Inadecuada atención en personas con enfermedades crónicas no transmisibles, en la Parroquia Urbana la Providencia, Machala - El Oro - Ecuador.

La labor educativa de promoción y de prevención, es un factor fundamental para enfrentar la problemática de las enfermedades crónicas no transmisibles. La intervención activa del personal de salud en la educación de los pacientes sobre la necesidad de mantener una adherencia terapéutica correcta es muy importante en la actualidad (Serra et al, 2018).

El estado nutricional de las personas, en especial, el estado de obesidad, es uno de los principales factores de riesgo para enfermedades cardiovasculares, que puede devenir en complicaciones como infartos de miocardio o eventos cerebrovasculares, principalmente, en pacientes que tienen enfermedades crónicas no transmisibles, como Hipertensión arterial y Diabetes Mellitus tipo 2, siendo necesario conocer si las estrategias de promoción que se realizan en los proyectos de vinculación ayudan a mejorar el estado nutricional de los participantes.

Por lo expuesto anteriormente, el objetivo del presente estudio es determinar la mejora nutricional, en los participantes del proyecto de Vinculación con la Sociedad: "Centro de Promoción y Prevención de la Salud Familiar "CEUPROPSF”.

\section{Materiales y Métodos}

Dentro del proyecto CEUPROPSF, se realizó el diagnóstico clínico de los habitantes de la Parroquia La Providencia, de los Barrios 10 de agosto, La Providencia, San Vicente, Cristóbal 
Colón y 16 de octubre, por medio de historias clínicas y posteriores seguimientos domiciliarios. Se realizaron estrategias de promoción y prevención como charlas, jornadas médicas, para lograr la concienciación de las causas y factores de riesgo de las enfermedades crónicas no transmisibles y mejorar la calidad de vida de los participantes del proyecto. Además, se dictaron charlas sobre nutrición y se realizaron actividades de actividad física.

Se realizó un estudio transversal descriptivo, observacional, no experimental, usando el método deductivo, cualitativo, que por sus características y delimitaciones permitió describir las variables en una sola medición.

La población de estudio fueron los pobladores adultos, en un rango de edad de 40 a 80 años, de la Parroquia La Providencia que padecían Diabetes Mellitus tipo 2 (DMT2), Hipertensión Arterial (HTA) o ambas patologías (HTA + DMT2), que pertenecía eran participantes del Proyecto CEUPROPSF, con un total de 57 participantes.

\section{Criterios de Inclusión y Exclusión}

Se incluyó adultos en un rango de 40 a 80 años, hombres y mujeres que padecían DMT2, HTA o ambas patologías.

Se excluyó a quienes no desearan participar, presenten alguna discapacidad para la comunicación verbal, no hayan firmado el consentimiento informado, no consuman medicamentos o no padezcan de DMT2 o HTA

\section{Procedimiento de Recolección de Datos}

Se recolectaron los datos, en las historias clínicas personales, con seguimientos quincenales. Se tomaron las medidas antropológicas como peso y talla, al inicio del programa y al finalizar el mismo. Para la evaluación de estado nutricional calculó el IMC, con el peso y talla de los participantes, clasificando a los participantes de la siguiente manera: Bajo peso $=$ IMC $<18.5 \mathrm{~kg} / \mathrm{m}^{2}$; peso normal = IMC de 18.5 a 24.9 $\mathrm{kg} / \mathrm{m}^{2} ; \quad$ sobrepeso IMC 25 a $29.9 \mathrm{~kg} / \mathrm{m}^{2}$; Obesidad moderada $=$ IMC $30-34.9 \mathrm{~kg} / \mathrm{m}^{2}$; Obesidad severa IMC $35-39.9 \mathrm{~kg} / \mathrm{m}^{2} ; \mathrm{y}, \mathrm{IMC} \geq 40 \mathrm{~kg} / \mathrm{m}^{2}$.

Formula cálculo IMC

$$
I M C=\frac{\operatorname{peso}(\mathrm{Kg})}{\operatorname{altura}^{2}(\mathrm{~m})}
$$

\section{Estrategias para Análisis de Datos}

Los datos fueron capturados y registrados en una base de datos. Para la tabulación de los resultados en la base de datos se utilizó el programa Microsoft Excel 2018. Para el cumplimiento de los objetivos se obtuvo la estadística descriptiva; para las variables categóricas: frecuencias y porcentajes. Para la realización del estudio se contó con el 
consentimiento informado de los participantes del proyecto, previo a la recolección de los datos.

\section{Resultados y Discusión}

La población objeto del estudio fueron 57 participantes del Proyecto Ceupropsf, de sexo femenino y masculino, que padecían de enfermedades crónicas no trasmisibles (Diabetes, Hipertensión Arterial o ambas.

Tabla 1.

Sexo de los participantes

\begin{tabular}{|c|c|c|c|c|c|c|}
\hline \multirow{2}{*}{ EDAD } & \multicolumn{2}{|c|}{ HOMBRE } & \multicolumn{2}{c|}{ MUJER } & \multicolumn{2}{c|}{ TOTAL } \\
\cline { 2 - 7 } & Frecuencia & Porcentaje & Frecuencia & Porcentaje & Frecuencia & Porcentaje \\
\hline $41-50$ años & 0 & $0 \%$ & 5 & $9 \%$ & 5 & $9 \%$ \\
\hline $51-60$ años & 6 & $11 \%$ & 9 & $16 \%$ & 15 & $26 \%$ \\
\hline $61-70$ años & 8 & $14 \%$ & 11 & $19 \%$ & 19 & $33 \%$ \\
\hline$>71$ años & 6 & $11 \%$ & 12 & $21 \%$ & 18 & $32 \%$ \\
\hline TOTAL & 20 & $35 \%$ & 37 & $65 \%$ & 57 & $100 \%$ \\
\hline
\end{tabular}

Fuente: Base de Datos Proyecto Ceupropsf

Los participantes estuvieron en el rango de 40 a 79 años, el promedio de edad fue de 60 años \pm 6. El rango de edad con mayor prevalencia fue del 61-70 años. En relación al sexo, el femenino fue el prevalente con $65 \%$. En concordancia con el estudio realizado por Miguel et al. (2017), sobre prevalencia de enfermedades crónicas no transmisibles y factores de riesgo en adultos mayores de Holguín, en donde el 85.9\%, fueron adultos mayores, siendo el sexo femenino el de mayor prevalencia $58.7 \%$.

Tabla 1.

Patologías por sexo

\begin{tabular}{|c|c|c|c|c|c|c|}
\hline \multirow{2}{*}{ Patología } & \multicolumn{2}{|c|}{ Hombre } & \multicolumn{2}{|c|}{ Mujer } & \multicolumn{2}{|c|}{ Total } \\
\hline & Frecuencia & Porcentaje & Frecuencia & Porcentaje & Frecuencia & Porcentaje \\
\hline HTA & 9 & $16 \%$ & 27 & $47 \%$ & 36 & $63 \%$ \\
\hline DMT2 & 4 & $7 \%$ & 5 & $9 \%$ & 9 & $16 \%$ \\
\hline HTA + DMT2 & 7 & $12 \%$ & 5 & $9 \%$ & 12 & $21 \%$ \\
\hline TOTAL & 20 & $35 \%$ & 37 & $65 \%$ & 57 & $100 \%$ \\
\hline
\end{tabular}

Fuente: Base de Datos Proyecto Ceupropsf

De los 57 participantes, 9 hombres y 27 mujeres tenían HTA (63\%), 4 hombres y 5 mujeres tenían DMT2 (16\%) y 7 hombres y 5 mujeres tenían DMT2 + HTA (21\%). Siendo el sexo femenino el de mayor prevalencia en DMT2 o HTA, mientras que los hombres tenían mayor prevalencia en padecer de ambas patologías (HTA+ DMT2). Se observa que en general, la enfermedad con mayor prevalencia fue la Hipertensión Arterial, datos concordantes con el artículo publicado por Buriticá y Ordóñez (2019), en donde la Hipertensión arterial fue la enfermedad crónica no transmisible con mayor índice de prevalencia $60.7 \%$ en la población adulta, seguida por la Diabetes Mellitus con $18.5 \%$. 


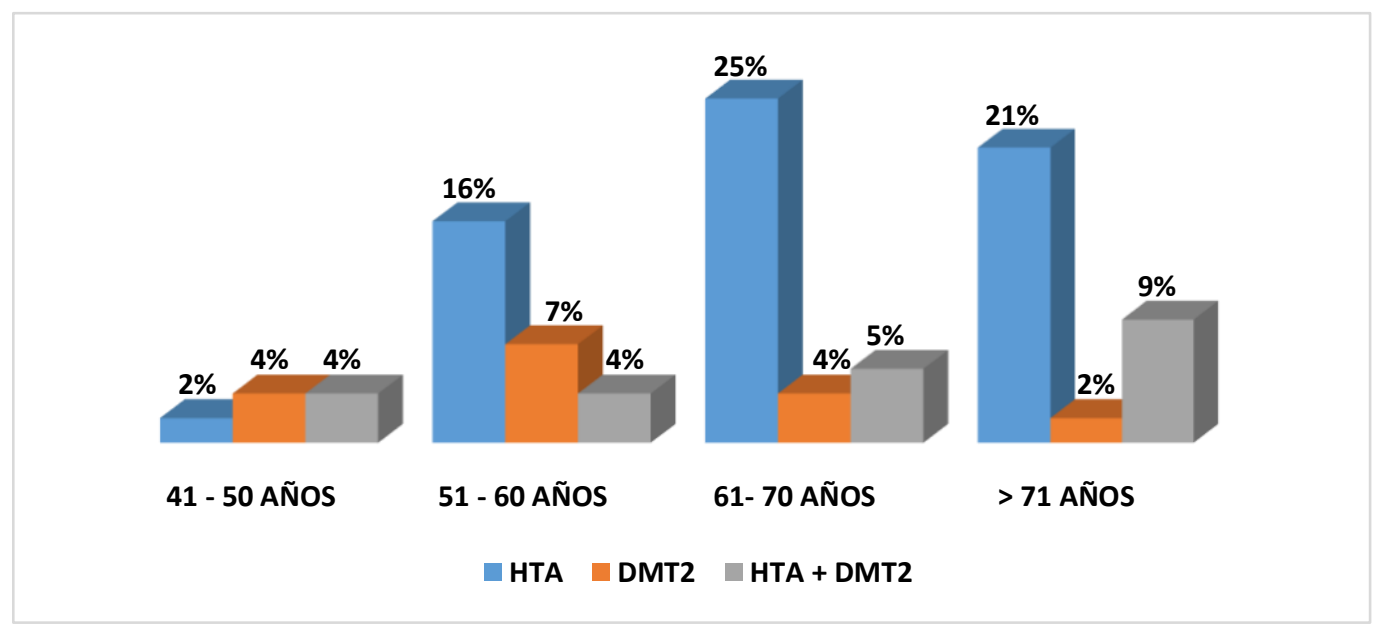

Figura 1.

Patologías según rango de edad

Fuente: Base de Datos Proyecto Ceupropsf

El grupo etario con mayor prevalencia de HTA fue de 61 a 70 años con el 25\%; en relación a la DMT2, el grupo etario prevalente fue el de 51 a 60 años, con el 7\%; y, el rango edad con prevalencia de HTA + DMT2, fue el >71 años 9\%. Evidenciándose que a mayor edad, existe mayor número de personas con enfermedades crónicas no transmisibles.

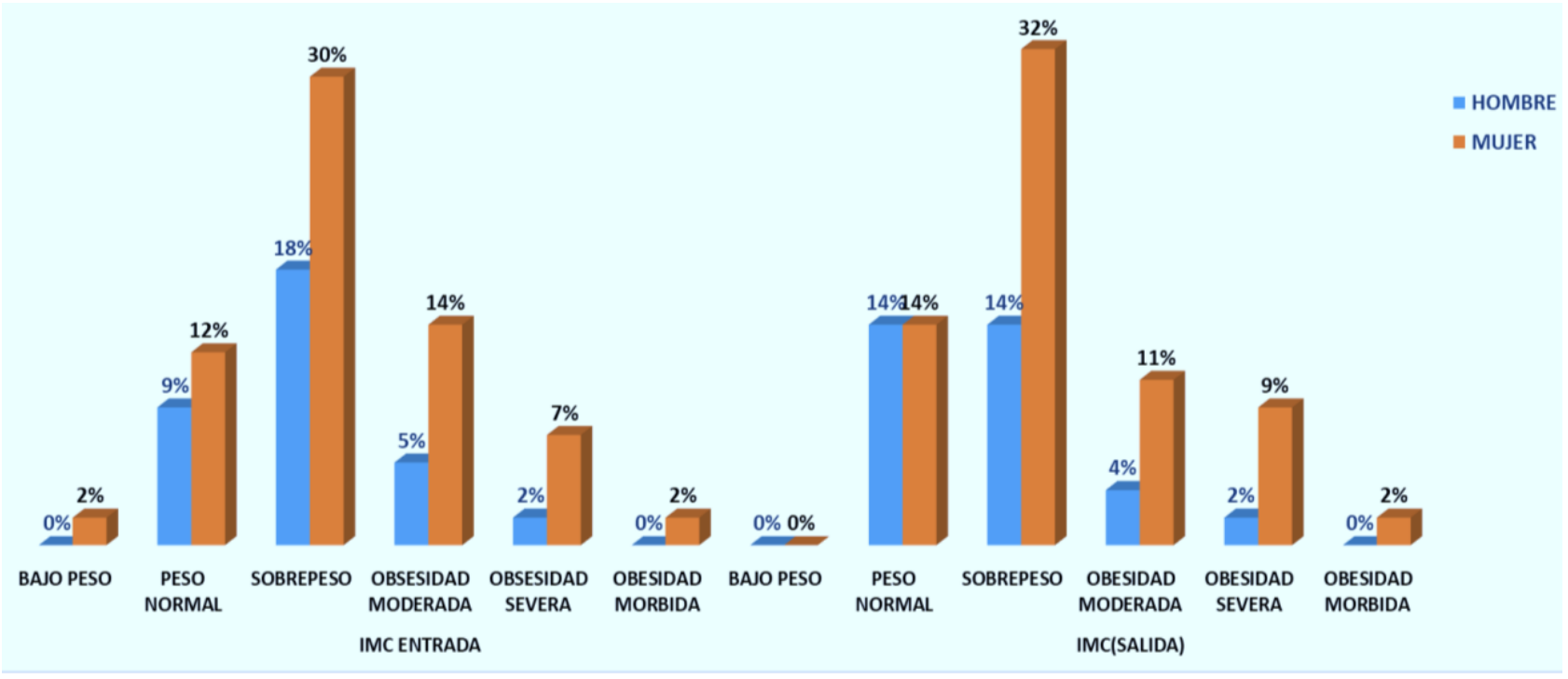

\section{Figura 2.}

Estado Nutricional al Inicio y término del Proyecto, según IMC

Fuente: Base de Datos Proyecto Ceupropsf

En relación al IMC al inicio el proyecto, $48 \%$ tenían sobrepeso, el $21 \%$ se encontraban con peso normal, el $19 \%$ tenían obesidad moderada, el $9 \%$ obesidad severa y el $2 \%$ obesidad mórbida y bajo peso. Al finalizar el proyecto, el $46 \%$ tenían sobrepeso, el $28 \%$ estaban en peso normal, el $15 \%$ en obesidad moderada, el $11 \%$ obesidad severa y el $2 \%$ obesidad mórbida, sin encontrarse participantes con peso bajo. En contraposición con el 
estudio realizado por Choque et al (2017), en donde los adultos mayores tenían el $24.3 \%$ de los participantes del estudio tenían bajo peso, el $51.3 \%$ tenían peso normal, el $5.1 \%$ tenían sobrepeso y el 19.2 presentaban obesidad, presentando la mayoría un estado de salud aceptable.

\section{Conclusiones}

La enfermedad prevalente entre los participantes del proyecto era la Hipertensión arterial, existiendo mayores participantes en el rango de edad de 60 a 71 años. Se evidencia una mejora considerable en el estado nutricional de los participantes de proyecto de vinculación CEUPROPSF, debido al aumento de peso en las personas que se encontraban bajo peso. Además, de la disminución de peso en las personas que se encontraban en obesidad severa y moderada. Las personas en peso normal se mantuvieron en su peso, evidenciándose que las estrategias de promoción y prevención instauradas en el Proyecto, han sido adecuadas y eficaces.

\section{Referencias}

Ángel, M y Valdés, S. (2016). Las enfermedades crónicas no transmisibles: una mirada actual ante el reto. Revista Finlay, 6(2):167-69. Disponible en: http://scielo.sld.cu/scielo.php?script=sci_arttext\&pid=S2221-24342016000200009

Buriticá, E., y Ordóñez, L. (2019). Implementación de medidas preventivas para enfermedades crónicas no transmisibles en la ciudad de Cali 2012-2014. Archivos Venezolanos de Farmacología y Terapéutica, 38(4), 391-395. Disponible en: https://www.revistaavft.com/images/revistas/2019/avft_4_2019/1_implementacion.pdf

Choque, et al (2017) Estado nutricional de los adultos mayores que asisten a la Universidad Municipal del adulto mayor. Revista "Cuadernos", 58(1)19-24. Disponible en: http://www.scielo.org.bo/scielo.php?script=sci_abstract\&pid=S165267762017000100003\&lng=es\&nrm=iss

Domínguez, et al. (2017) Las medidas antropométricas como indicadores predictivos de riesgo metabólico en una población mexicana. Nutrición Hospitalaria, 34(1):96-101. Disponible en: https://scielo.isciii.es/scielo.php?script=sci_abstract\&pid=S0212$\underline{16112017000100015}$

García, J., et al. (2018) Nuevo enfoque de la nutrición. Valoración del estado nutricional del paciente: función y composición corporal. Nutrición Hospitalaria. 35 (3): 1-14. Disponible en: http://dx.doi.org/10.20960/nh.2027

García, A. y Creus E. (2016) La obesidad como factor de riesgo, sus determinantes y tratamiento. Revista Cubana de Medicina General Integral, 32(3) 1-13. Disponible en: http://scielo.sld.cu/scielo.php?script=sci_arttext\&pid=S0864-

21252016000300011\&lng=es\&tlng=es.

Hernández, et al. (2018) Utilidad del índice cintura/cadera en la detección del riesgo cardiometabólico. Revista Cubana de Endocrinología, 29(2):1-16. 
Instituto Nacional de Estadísticas y Censos (2017) Camas y Egresos Hospitalarios 2017. Disponible en: https://www.ecuadorencifras.gob.ec/camas-y-egresos-hospitalarios$\underline{2017}$

Miguel et al. (2017) Prevalencia de enfermedades crónicas no transmisibles y factores de riesgo en adultos mayores de Holguín. Revista Finlay, 7(3), 155-167. Disponible en: http://scielo.sld.cu/scielo.php?script=sci_arttext\&pid=S222124342017000300002\&lng=es\&tlng=es

Moreno, M. (2012) Definición y clasificación de la obesidad. Revista Médica Clínica Las Condes, 23 (2) 124-128. Disponible en: https://doi.org/10.1016/S07168640(12)70288-2.

Organización Mundial de la Salud, OMS. (2018). Enfermedades no transmisibles. Disponible en: https://www.who.int/es/news-room/fact-sheets/detail/noncommunicable-diseases.

Organización Panamericana de la Salud, OPS (2017) Día Mundial de la Hipertensión 2017: Conoce tus números. Recuperado de: https://www.paho.org/hq/index.php?option=com_content\&view=article\&id=13257:diamundial-de-la-hipertension-2017-conoce-tus-numeros\&Itemid=42345\&lang=es.

Organización Panamericana de la Salud, OPS. (2018) Día Mundial de la Diabetes 2018. Disponible en: https://www.paho.org/hq/index.php?option=com_content\&view=article\&id=14780:w orld-diabetes-day-2018-diabetes-concerns-every-family\&Itemid=1969\&lang=es.

Nafxiel R. y Alcázar C. (2011) Obesidad y riesgo cardiometabólico. Revisión. CIMEL Ciencia e Investigación Médica Estudiantil Latinoamericana. 16(2):106-13. Disponible en: http://www.fepreva.org/curso/5to_curso/bibliografia/volumen1/u4_vol1_obesidad.pdf Serra, et al. (2018). Las enfermedades crónicas no transmisibles: magnitud actual y tendencias futuras. Revista Finlay. 8(2):140-48. Disponible en: http://scielo.sld.cu/scielo.php?script=sci_arttext\&pid=S222124342018000200008\&lng=es\&tlng=es. 